\title{
$\varepsilon$-carbide in Alloy Steels: First-principles Assessment
}

\author{
Jae Hoon Jang ${ }^{a}$ In Gee Kim ${ }^{a}$ H. K. D. H. Bhadeshia ${ }^{a, b}$ \\ ${ }^{a}$ Graduate Institute of Ferrous Technology, Pohang University of Science and \\ Technology, Pohang 790-784, Republic of Korea \\ ${ }^{\mathrm{b}}$ Department of Materials Science and Metallurgy, University of Cambridge, \\ Cambridge CB2 3QZ, U.K.
}

\begin{abstract}
There is now a large volume of sophisticated steels which rely on silicon as an alloying addition with the purpose of avoiding the precipitation of cementite. But there is also evidence that the silicon can enhance the formation of $\varepsilon$-carbide; the mechanism of this effect is not understood and the absence of appropriate thermodynamic data makes it impossible to conduct calculations. We report here some ab initio calculations which throw light on both of these issues and suggest novel experiments.
\end{abstract}

Key words: Steels, $\varepsilon$-carbide, cementite, silicon, tempering

There are two long-established reasons for adding silicon into certain highperformance steels. The first is in the context of steels which are cheap and yet contain considerable quantities of austenite, which permits some of the plasticity needed to sustain deformation via martensitic transformation [1-5]. These TRIP-assisted steels rely on the fact that silicon retards the precipitation of cementite from austenite, because at the temperatures of interest, the silicon is forced into the cementite lattice and hence dramatically reduces its stability. As a consequence, the carbon that is partitioned during the formation of ferrite enriches and stabilises a proportion of the austenite to ambient temperatures.

The second is in the manufacture of extremely strong alloys based on the tempered martensite microstructure, where a retardation of cementite permits more controlled heat-treatments in regimes where embrittlement phenomena are absent $[6,7]$.

Email address: igkim@postech.ac.kr (In Gee Kim). 
It would, in both cases, be useful to know the minimum amount of silicon that needs to be added in order to generate the appropriate microstructure. It is also necessary to optimise the silicon concentration to mitigate the formation of adherent red-scale on the surface of the steel, which interferes with the ability to produce aesthetically appealing products [8]. All this requires a knowledge of the thermodynamics of silicon in cementite, which cannot be accessed experimentally because of the incredibly low solubility of silicon in this phase. There has, nevertheless, been significant progress with a variety of $a b$ initio calculations of thermodynamic parameters $[9,10]$, some of which are now implemented in phase diagram estimations [11].

One remaining difficulty is that there is circumstantial experimental evidence to show that the precipitation of the transition $\varepsilon$-carbide is enhanced in the presence of silicon, whether this is from supersaturated austenite [12] or martensite $[6,7]$. There is some confusion as to whether the $\varepsilon$ can form in austenite - experiments suggest that it can [12] but calculations contradict this [13], although the reliability of the assumed thermodynamic data is not clear in the latter case. The mechanism for the possible enhancement of $\varepsilon$ is not understood, but it could be the case that $\varepsilon$ is more tolerant to silicon than cementite or that it becomes prominent simply because cementite has been suppressed. The $\varepsilon$ [14], like cementite [15], has been shown to inherit the silicon concentration of the steel during precipitation at relatively low temperatures; the redistribution of silicon during the transition of $\varepsilon$ to cementite has also been reported [9]. The goal of the work presented here was to determine by first-principle calculations, the role of silicon on the thermodynamics and mechanism of $\varepsilon$-carbide precipitation.

$\varepsilon$-carbide is hexagonal $P 6_{3} 22$ with lattice parameters $a_{h}=2.752 \AA$ and $c_{h}=$ $4.353 \AA$ [16], containing two Fe atoms, Fig. 1 . The conventional cell with $a=$ $\sqrt{3} a_{h}$ and $c=2 c_{h}$ has 6 Fe atoms and on average 2-3 carbon atoms in order to achieve the formula $\mathrm{Fe}_{2.4} \mathrm{C}$. Note that substitution of $\mathrm{Fe}$ by a single $\mathrm{Si}, \mathrm{Al}$ or $\mathrm{Mn}$ atom into the cell illustrated in Fig. 1b would lead to concentrations of $4.00,3.85$ and 7.53 at\%, respectively. Because of symmetry, calculations only are necessary for substitution into layers 1 or 2 in Fig. 1 b.

The Kohn-Sham equation was solved self-consistently in terms of the total energy all-electron full-potential linearised augmented plane-wave (FLAPW) method [17, 18] implemented in the QMD-FLAPW algorithm [19] with the generalized gradient approximation [20]. An energy cutoff at 21 Ry was employed for the linearized augmented plane wave basis set. A 340 Ry cutoff was used for the star-functions depicting the charge density and potential in the interstitial region. Lattice harmonics with $l \leq 8$ were employed to expand the charge density, potential and wave-functions inside each muffin-tin sphere of radii 2.04 a.u. for $\mathrm{Fe}, \mathrm{Si}, \mathrm{Al}$ and $\mathrm{Mn}$, and $1.30 \mathrm{a}$.u. for $\mathrm{C}$ atoms. The chosen the computational parameters satisfy the convergence test [21]. Improved 
tetrahedron method [22] for the Brillouin zone integrations was performed on a $9 \times 9 \times 9$ Monkhorst-Pack mesh [23]. All core electrons were treated fully relativistically and valence state scalar relativistically with the explicit orthogonalization method [24]. Self-consistency was assumed when the difference between the input and output charge and spin densities is less than $10^{-4}$ electrons a.u. ${ }^{-3}$ Beginning with the reported lattice parameters [16], 25 combinations of $a$ and $c$ varying by $\pm 2 \%$ in steps of $1 \%$ were used as pivot points. The minimum energy point determined along $a$ was then used to obtain an optimal value of $c$, and the procedure was iterated until an error within $0.2 \%$ was achieved. We applied the multistage lattice parameter optimization scheme [10] with the fourth order parameter fitting [25]. Atomic relaxation at each pivot point was achieved using the total energy and force minimisation [26], until the force on each atom is less than $2 \mathrm{mRya}^{-u^{-1}}$ and displacement $3 \times 10^{-3}$ a.u.

Calculated equilibrium lattice parameters for $0 \mathrm{~K}$ are listed in Table 1 together with ambient temperature experimental data from [16]. The difference between the two sets of data is $0.6 \%$ and $0.9 \%$ for $a$ and $c$ respectively, and as might be expected, the ambient temperature values are larger. Data for the temperature dependence of the $c_{h} / a_{h}$ ratio (though not of the individual parameters) are available [16]; extrapolation to $0 \mathrm{~K}$ gives 1.575 , which is only $0.1 \%$ smaller than calculated. Note that there is a mistake in Ref. [16] where the units of temperature in the graph are incorrectly stated as ${ }^{\circ} \mathrm{C}$ instead of Kelvin. Unlike $\mathrm{Al}$ and $\mathrm{Mn}$, the substitution of Si leads to a noticeable decrease of $0.5 \%$ in the $c$-parameter whereas the change in $a$ is within the limits of numerical precision. The implications here will be discussed later.

Somewhat surprising results are listed in Table 2, where the formation energy with integers $l, m$ and $n$ is given by

$$
\Delta U=E\left(\mathrm{Fe}_{l} \mathrm{C}_{m} X_{n}\right)-l E(\mathrm{Fe})-m E(\mathrm{C})-n E(X)
$$

where $E\left(\mathrm{Fe}_{l} \mathrm{C}_{m} X_{n}\right), E(\mathrm{Fe})$ and $E(\mathrm{C})$ represent the total energies, at the corresponding equilibrium lattice parameters, of ferromagnetic body-centred cubic lattices and of graphite respectively, and $X$ is either $\mathrm{Si}, \mathrm{Al}$ or $\mathrm{Mn}$. $E(X)$ is the total energy of $\mathrm{Si}, \mathrm{Al}$ or $\mathrm{Mn}$ at the corresponding equilibrium lattice constants of the diamond, face-centred cubic and body-centred cubic structures, respectively.

It appears from Table 2 that it is even less favourable to substitute silicon into $\varepsilon$ than in cementite. The result firmly establishes that relative to cementite, any enhancement of $\varepsilon$ precipitation when silicon is incorporated into its lattice cannot be a thermodynamic effect. This is somewhat inconsistent with the qualitative experimental observations reported in the literature. Therefore, possible consequences which are kinetic in origin were investigated. 
The orientation relationship between $\varepsilon$ and martensite $\left(\alpha^{\prime}\right)$ is as follows [27, 28]:

$$
(101)_{\alpha^{\prime}}\left\|(10 \overline{1} 1)_{\varepsilon} \quad(011)_{\alpha^{\prime}}\right\|(0001)_{\varepsilon} \quad[11 \overline{1}] \|[1 \overline{2} 10]_{\varepsilon}
$$

Using the lattice parameters $a_{\alpha^{\prime}}=2.85 \AA$ and $c_{\alpha^{\prime}}=2.98 \AA$ for 1 at. $\%$ carbon martensite [29], it can be demonstrated that the spacing of the $[011]_{\alpha^{\prime}}$ and $[11 \overline{1}]_{\alpha^{\prime}}$ is smaller than corresponding directions in pure $\varepsilon$ by $9.2 \%$ and $4.7 \%$, respectively. Therefore, the addition of silicon which leads to a contraction along the $c$-axis by $0.5 \%$ should improve the coherency of the carbide with the martensite during the nucleation stage, and this may explain the apparent enhancement of this transition carbide relative to cementite when the steel is alloyed with silicon.

Both aluminium and manganese stabilise $\varepsilon$ more than they do cementite (Table 2). This actually leads to a lower formation energy for $\varepsilon$ than cementite in the case of manganese substitution and opens up the possibility of the $\varepsilon$ becoming the stable precipitate in Mn-rich steels.

In summary, the work suggests two critical experiments, a quantitative study of the relative kinetics of $\varepsilon$-carbide and cementite during the tempering of martensite, as a function of the silicon concentration, and with a focus on nucleation rate. Secondly, an investigation of Mn-rich steels (with concentrations in excess of $7.5 \mathrm{wt} \%$ ) to see if the relative stabilities of the two carbides are reversed.

This work was supported by the Steel Innovation Programme by POSCO and the World Class University programme (Project No. R32-2008-000-10147-0) by the National Research Foundation of Korea.

\section{References}

[1] O. Matsumura, Y. Sakuma, H. Takechi: Transactions of the Iron and Steel Institute of Japan 27 (1987) 570-579.

[2] O. Matsumura, Y. Sakuma, H. Takechi: Scripta Metallurgica 27 (1987) 1301-1306.

[3] P. J. Jacques: Current Opinion in Solid State and Materials Science 8 (2004) 259-265.

[4] B. De Cooman: Current Opinion in Solid State and Materials Science 8 (2004) 285-303.

[5] H. K. D. H. Bhadeshia: Proceedings of the Royal Society of London A 466 (2010) 3-18.

[6] W. M. Garrison Jr.: Journal of Metals 42 (1990) 20-24. 
[7] F. B. Pickering: Physical Metallurgy and the Design of Steels: Applied Science Publishers, Essex, U. K., 1978.

[8] R. K. S. Raman: Engineering Failure Analysis 13 (2006) 1044-1050.

[9] G. Miyamoto, J. C. Oh, K. Hono, T. Furuhara, T. Maki: Acta Materialia 55 (2007) 5027-5038.

[10] J. H. Jang, I. G. Kim, H. K. D. H. Bhadeshia: Computational Materials Science 44 (2009) 1319-1326.

[11] J. H. Jang, I. G. Kim, H. K. D. H. Bhadeshia: Materials Science Forum 638-642 (2010) 3319-3324.

[12] F. C. I. Gutierrez, J. Aranzabal, J. J. Urcola: Metallurgical \& Materials Transactions A 26 (1995) 1045-1060.

[13] N. G. Shaposhnikov, B. M. Mogutnov: Russian Metallurgy (Metally) 2008 (2008) 174-179.

[14] S. J. Barnard and G. D. W. Smith and A. J. Garratt-Reed and J. Vander Sande: Solid-Solid Phase Transformations, TMS-AIME, Materials Park, Ohio, (1981) 881-885.

[15] S. S. Babu and K. Hono and T. Sakuri: Metallurgical and Materials Transactions A 25 (1994) 499-508.

[16] S. Nagakura: Journal of the Physical Society of Japan 14 (1959) 186-195.

[17] E. Wimmer, H. Krakauer, M. Weinert, A. J. Freeman: Physical Review B 24 (1981) 864-875.

[18] M. Weinert, E. Wimmer, A. J. Freeman: Physical Review B 26 (1982) 4571-4578.

[19] http://www.flapw.com.

[20] J. P. Perdew, K. Burke, M. Ernzerhof: Physical Review Letters 77 (1996) 3865-3868.

[21] S. -W. Seo, Y. -Y. Song, G. Rahman, I. G. Kim, M. Weinert, A. J. Freeman: Journal of Magnetics 14 (2009) 137.

[22] J. -H. Lee, T. Shishidou, A. J. Freeman: Physical Review B 66 (2002) 233102.

[23] H. J. Monkhorst, J. D. Pack: Physical Review B 13 (1976) 3865-3868.

[24] M. Weinert, G. Schneider, R. Podloucky, J. Redinger: Journal of Physics: Condensed Matter 21 (2009) 084210.

[25] J. C. Boettger: Physical Review B 55 (1997) 11202-11212.

[26] W. Mannstadt, A.J. Freeman: Physical Review B 55 (1997) 13298.

[27] K. H. Jack: Journal of the Iron and Steel Institute 169 (1951) 26-36.

[28] H. K. D. H. Bhadeshia: Bainite in Steels, 2nd edition: Institute of Materials, London, 2001.

[29] C. S. Roberts: Trans. AIME 197 (1953) 203-204. 
Table 1

Measured and calculated values of lattice parameters of pure, $\mathrm{Si}, \mathrm{Al}$ and $\mathrm{Mn}$ substituted $\varepsilon$-carbide. Values in parentheses represent percent deviation relative to pure

\begin{tabular}{lccc} 
E. & $a / \AA$ & $c / \AA$ & $c_{h} / a_{h}$ \\
\hline Measured [16] & 4.767 & 8.708 & 1.582 \\
Calculated, pure $\varepsilon$ & 4.740 & 8.631 & 1.577 \\
Si substituted & $4.730(-0.2 \%)$ & $8.590(-0.5 \%)$ & 1.573 \\
Al substituted & $4.742(+0.0 \%)$ & $8.685(+0.6 \%)$ & 1.586 \\
Mn substituted & $4.738(-0.1 \%)$ & $8.664(+0.4 \%)$ & 1.584 \\
\hline
\end{tabular}

Table 2

Formation energies (kJ per mole of unit cells containing 12 Fe atoms; quantities for cementite from [10]). Values in parentheses represent difference relative to pure carbide. In the case of $\varepsilon$, only the values for $\mathrm{Si}$ and $\mathrm{Al}$ in layer I and $\mathrm{Mn}$ in layer II are fully relaxed. The other sites are not favoured, to an extent well beyond the changes expected on relaxation.

\begin{tabular}{lcccc}
\hline & \multicolumn{2}{c}{$\varepsilon$-carbide } & \multicolumn{2}{c}{ Cemeniteite } \\
System & Layer I & Layer II & $\mathrm{Fe}(4 \mathrm{c})$ & $\mathrm{Fe}(8 \mathrm{~d})$ \\
\hline Pure carbide & 106.0 & 106.0 & 86.1 & 86.1 \\
Si substituted & $154.4(+48.4)$ & 236.4 & 138.1 & $123.2(+37.1)$ \\
Al substituted & $84.7(-21.3)$ & 178.7 & 76.1 & $72.5(-13.6)$ \\
Mn substituted & 102.3 & $74.8(-31.2)$ & 81.7 & $81.1(-5.0)$ \\
\hline
\end{tabular}



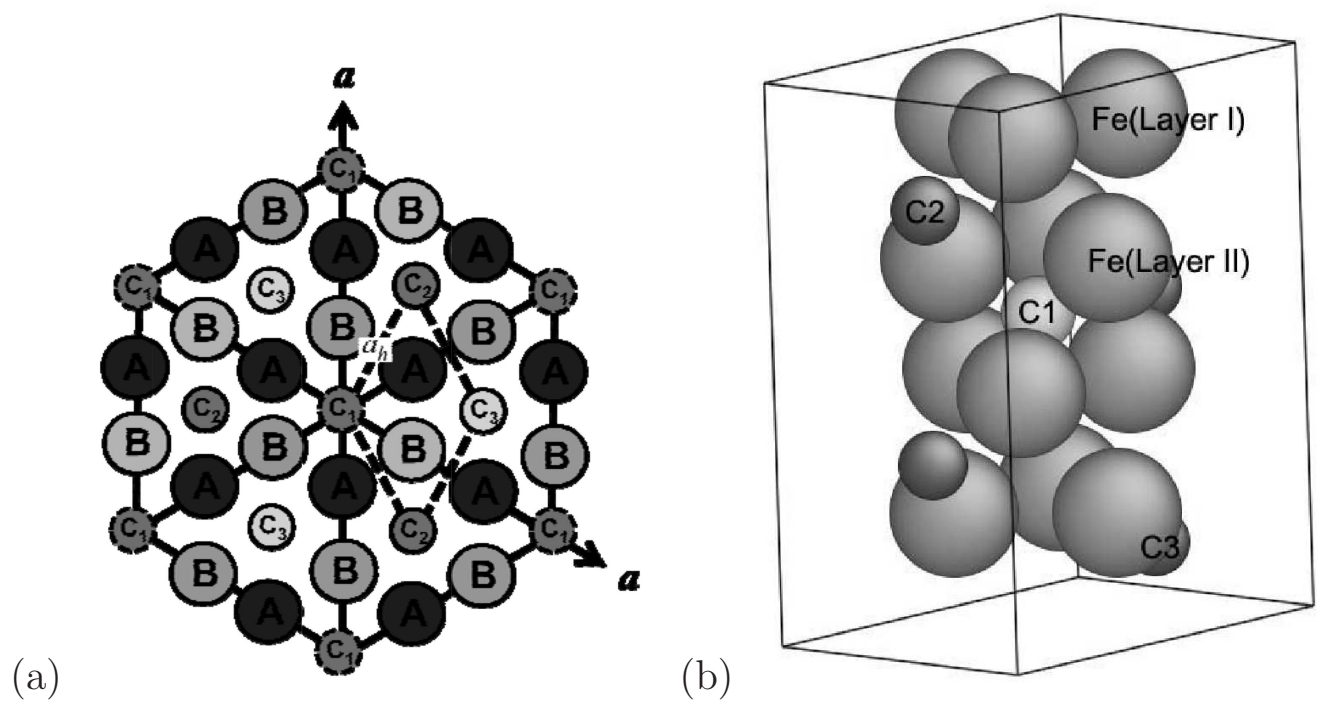

Fig. 1. (a) Projection on basal plane. The iron atoms are located at $\mathrm{A}(z=0)$ and $\mathrm{B}\left(z=\frac{1}{2}\right)$ where $z$ refers to the fractional height. The carbon atoms may occupy $\mathrm{C}_{1}, \mathrm{C}_{2}, \mathrm{C}_{3}$ at $z=\frac{1}{4}, \frac{1}{4}, \frac{3}{4}$ respectively [16]. (b) $\mathrm{Fe}_{2.4} \mathrm{C}$ where the $\mathrm{C}_{1}$ is partially occupied. 